\title{
The effect of red blood cell transfusion on plasma hepcidin and growth differentiation factor 15 in gastric cancer patients: a prospective study
}

\author{
Jingfu Liu ${ }^{1}$, Shan Chen ${ }^{1}$, Xianren Ye $e^{1,2}$ \\ ${ }^{1}$ Department of Blood Transfusion, Fujian Cancer Hospital \& Fujian Medical University Cancer Hospital, Fuzhou 350014, China; ${ }^{2}$ Fujian Provincial \\ Key Laboratory of Tumor Biotherapy, Fuzhou 350014, China \\ Contributions: (I) Conception and design: J Liu, X Ye; (II) Administrative support: X Ye; (III) Provision of study materials or patients: J Liu, S Chen; (IV) \\ Collection and assembly of data: S Chen; (V) Data analysis and interpretation: J Liu, X Ye; (VI) Manuscript writing: All authors; (VII) Final approval \\ of manuscript: All authors. \\ Correspondence to: Xianren Ye. Fuma Road, Jin'an District, Fuzhou 350014, China. Email: fjthy@sina.com.
}

\begin{abstract}
Background: Hepcidin and growth differentiation factor 15 (GDF-15) have been reported to be highly expressed in various cancers. Serum hepcidin and GDF-15 levels were demonstrated to be potential prognostic markers in cancers. This study aims to evaluate the effect of red blood cell (RBC) transfusion on plasma hepcidin and GDF-15 in gastric cancer patients.

Methods: In this prospective study, 40 patients with gastric cancer were eligible for this study. Peripheral blood samples were obtained before and within $24 \mathrm{~h}$ after RBC transfusion. A routine blood test was performed before transfusion and within $24 \mathrm{~h}$ post-transfusion. Plasma hepcidin, GDF-15, interleukin 6 (IL-6) and erythropoietin were determined by ELISA.

Results: In patients with metastasis, plasma hepcidin $(\mathrm{P}=0.02)$, and GDF-15 ( $\mathrm{P}=0.01)$ levels were higher than without metastasis. Plasma hepcidin was increased after RBC transfusion $(\mathrm{P}=0.001)$, while plasma erythropoietin was decreased after transfusion $(\mathrm{P}=0.03)$. However, $\mathrm{RBC}$ transfusion did not affect plasma GDF-15 ( $\mathrm{P}=0.32)$ and IL-6 (P=0.12). The effect of RBC transfusion on variables did not differ between metastatic and non-metastatic patients. The mean percentage change of hepcidin in transfusion volume 4 unit (U) was more than $2 \mathrm{U}$.
\end{abstract}

Conclusions: RBC transfusion could increase plasma hepcidin and have no effect on plasma GDF-15 in gastric patients.

Keywords: Red blood cell transfusion (RBC transfusion); hepcidin; growth differentiation factor 15 (GDF-15); gastric cancer

Submitted Apr 28, 2019. Accepted for publication Jul 24, 2019.

doi: $10.21037 /$ atm.2019.08.33

View this article at: http://dx.doi.org/10.21037/atm.2019.08.33

\section{Introduction}

The incidence and mortality of gastric cancer are the second highest among malignant tumors in China (1). Anemia is one of the most common complications in gastric cancer. In 2012, a survey from China reported that the highest incidence $(66.99 \%)$ of anemia was observed in upper gastrointestinal cancer patients among multiple solid cancers. Anemia is indicated to be associated with a reduction of the quality of life (2-4) and to be an adverse factor for treatment outcomes and long-term prognosis $(5,6)$ in cancer patients. Red blood cell $(\mathrm{RBC})$ transfusion is an option of treatment for anemia, especially for patients who need rapid improvement in hemoglobin level. However, RBC transfusion not only can transmit infectious disease but can also adversely impact post-operation complications $(7,8)$, cancer recurrence, and survival (9-12). 
The exact mechanism is still unclear, as most studies about the mechanism are focused on transfusion-related immunomodulation.

Hepcidin, a 25 -amino acid peptide primarily produced in the liver, is a key iron-regulatory hormone. Hepcidin regulates iron metabolism by inhibiting the function of its receptor ferroportin, the iron transporter expressed on the basolateral membrane of duodenal enterocytes (13). Hepcidin, which is also expressed by malignant cells (14-16), is elevated in many cancer patients (17). Previous studies have found increased serum hepcidin in non-small cell lung cancer (18) and breast cancer patients (19) rather than in healthy individuals or those with benign disease. High serum hepcidin in cancer patients has been associated with metastasis $(20,21)$, TNM stage, and poor prognosis (22). The possible explanation for this is that hepcidin degrades ferroportin-1 and results in the accumulation of iron in tumor cells $(17,23)$. High levels of inflammatory cytokines and bone morphogenetic proteins in cancer patients may account for high hepcidin levels $(18,24)$. The regulation of hepcidin can be affected by many factors, such as iron status (25), erythropoietic activity (26), inflammatory cytokines, hypoxia (27), and the synthesis of cancer cells in each patient.

Growth differentiation factor 15 (GDF-15), one of the transforming growth factor $\beta$ (TGF- $\beta$ ) superfamily members, was identified as one of the mediators of hepcidin expression (28). A positive correlation between GDF-15 and hepcidin has been verified in anemic patients (29). Additionally, GDF-15 is reported to be over-expressed in various cancers and is associated with the proliferation, metastasis, and prognosis (30), while a high level of GDF15 in cancer patients has been associated with cancer metastasis and poor overall survival (31).

Previous studies have reported that RBC transfusion could increase serum hepcidin in preterm infants (32) and patients with $\beta$-thalassemia (33). The explanation for this was that RBC transfusion increased the number of erythrocytes and down-regulated the production of erythromycin, which stimulated hepcidin production by the bone morphogenetic protein-SMAD pathway (13).

To date, there are no data concerning the effect of RBC transfusion on plasma hepcidin and GDF-15 in gastric cancer patients. We hypothesized that RBC transfusion could increase plasma hepcidin and GDF-15 in gastric cancer patients. Elevated plasma hepcidin and GDF-15 after transfusion may be associated with adverse effects on cancer outcomes from transfusion. The purpose of this

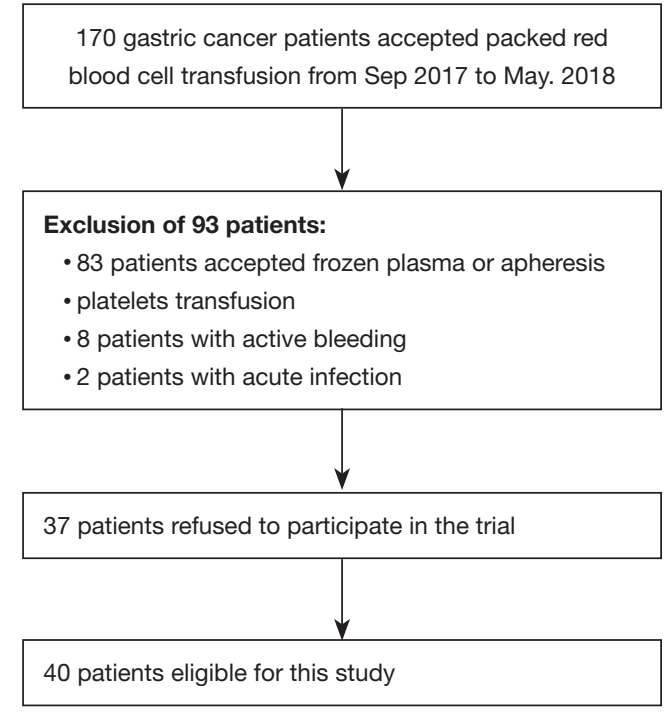

Figure 1 The process of patient enrollment.

study was to determine the change of plasma hepcidin and GDF-15 after RBC transfusion.

\section{Methods}

\section{Study design and patients selection}

A prospective study was conducted in Fujian Cancer Hospital from September of 2017 to May of 2018. The Ethical Committee of the Fujian Cancer Hospital approved this study. Patients meeting the following criteria were enrolled in this study: (I) confirmation of gastric cancer by pathologic or/and imaging examination; (II) adult patients; (III) patients who accepted packed RBC transfusion; (IV) blood routine test performed before transfusion and within $24 \mathrm{~h}$ post-transfusion. The exclusion criteria were as follows: (I) patients who accepted frozen plasma or apheresis platelets transfusion; (II) recent acute infection; (III) RBC transfusion refractoriness. The process of patient enrollment is shown in Figure 1.

\section{RBC transfusion}

The clinicians decided upon RBC transfusion according to our hospital transfusion guidelines. In principle, preoperative $\mathrm{RBC}$ is performed for asymptomatic patients if the hemoglobin level falls to $70 \mathrm{~g} / \mathrm{L}$ or intraoperative blood loss of $>1,000 \mathrm{~mL}$ occurs. Moreover, for medical patients, $\mathrm{RBC}$ transfusion is initiated before the hemoglobin 
Table 1 Characteristics and baseline variables

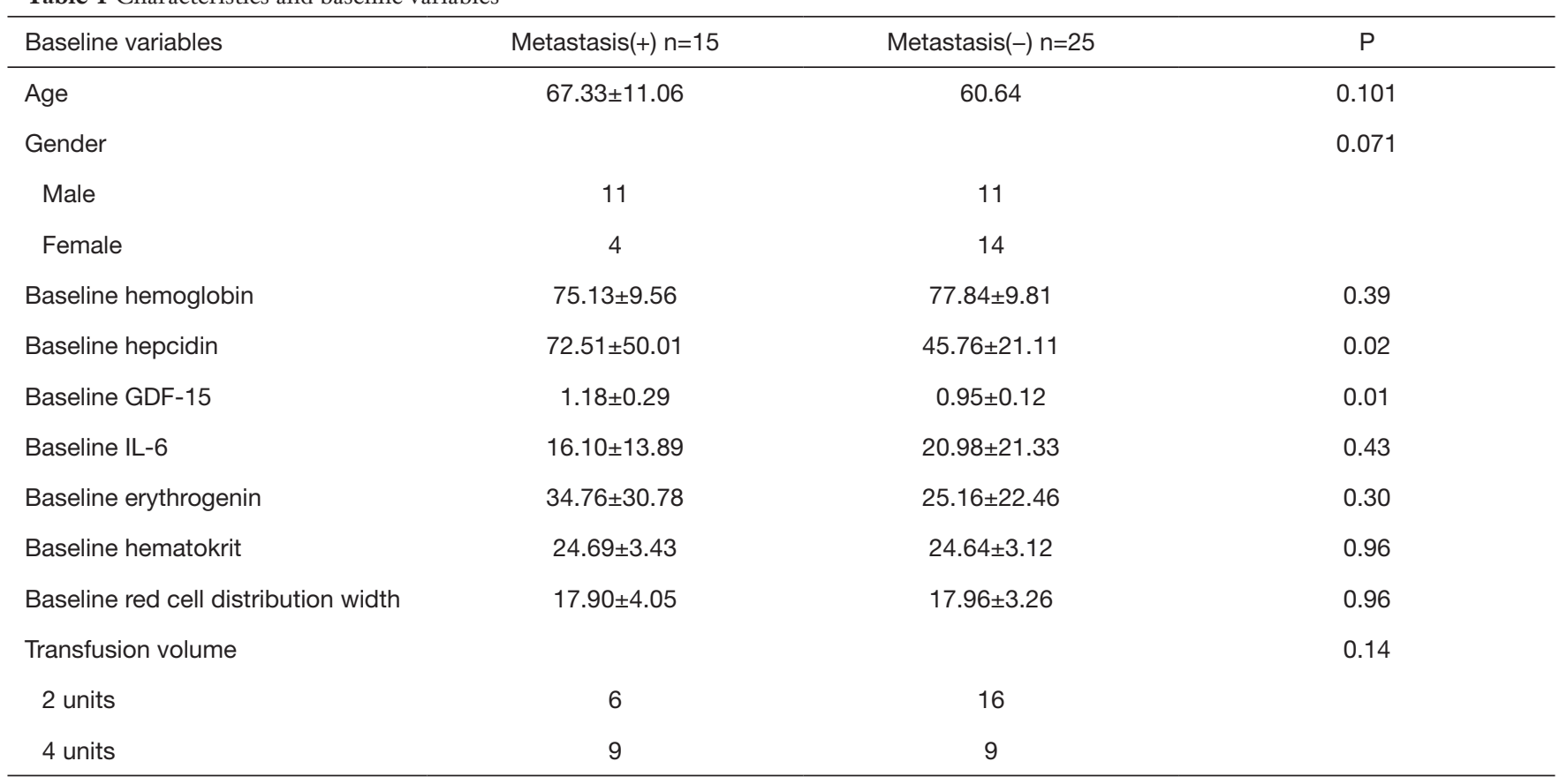

GDF-15, growth differentiation factor 15; IL-6, interleukin 6 .

concentration declines to $60 \mathrm{~g} / \mathrm{L}$ or hematocrit declines to 0.22 . Packed RBCs in this study were provided and produced by Fujian Blood Center. One unit (U) packed $\mathrm{RBC}$ was produced from $200 \mathrm{~mL}$ whole blood.

\section{Measurement of plasma variables}

Peripheral venous blood samples were drawn into tubes containing ethylenediamine tetraacetic acid (EDTA) on two time points: before transfusion and within $24 \mathrm{~h}$ posttransfusion. Samples were centrifuged at 3,400 rpm for $5 \mathrm{~min}$ at room temperature. The plasma was extracted and frozen below $-80{ }^{\circ} \mathrm{C}$ for use. Blood routine test was performed using the Sysmex hematology XE2100L analyzer (Sysmex, Tokyo, Japan). Commercial ELSIA kits were used to determine plasma hepcidin (R\&D, MN, USA), erythrogenin (Abcam, Cambridge, UK), interleukin 6 (IL-6) (Boster, Wuhan, China), and GDF-15 (Blue Gene, Shanghai, China). The clinical tumor stage was determined according to the 8th edition of the AJCC cancer staging manual.

\section{Statistical analysis}

All statistical analyses were performed using IBM SPSS version 23.0 (IBM Corporation, Chicago, IL, USA). Patients' characteristics were described by frequencies (percentages) and mean value. Variables were compared between pre- and post-transfusion by paired $t$-test. A P value of $<0.05$ was considered to indicate a statistically significant difference. Change (\%) was defined by the following formula: (post-transfusion value - pre-transfusion value)/pre-transfusion value $\times 100 \%$. Figures were drawn by GraphPad Prism 7 (GraphPad Software, La Jolla, CA, USA) and Office Visio.

\section{Results}

A total of 40 gastric cancer patients were enrolled in this study, among whom 15 were with metastasis, and 25 were non-metastasis. The process of patient selection is shown in Figure 1. Baseline characteristics are given in Table 1. Age, gender, baseline hemoglobin, IL-6, erythrogenin, hematocrit, and red cell distribution width (RDW) of patients were not statistically different between the metastasis(+) group and the metastasis(-) group. The levels of plasma hepcidin and GDF-15 of metastatic patients were higher than those in non-metastatic patients.

Figure 2 summarizes the changes in the percentage (\%) of plasma variables between pre-transfusion and 

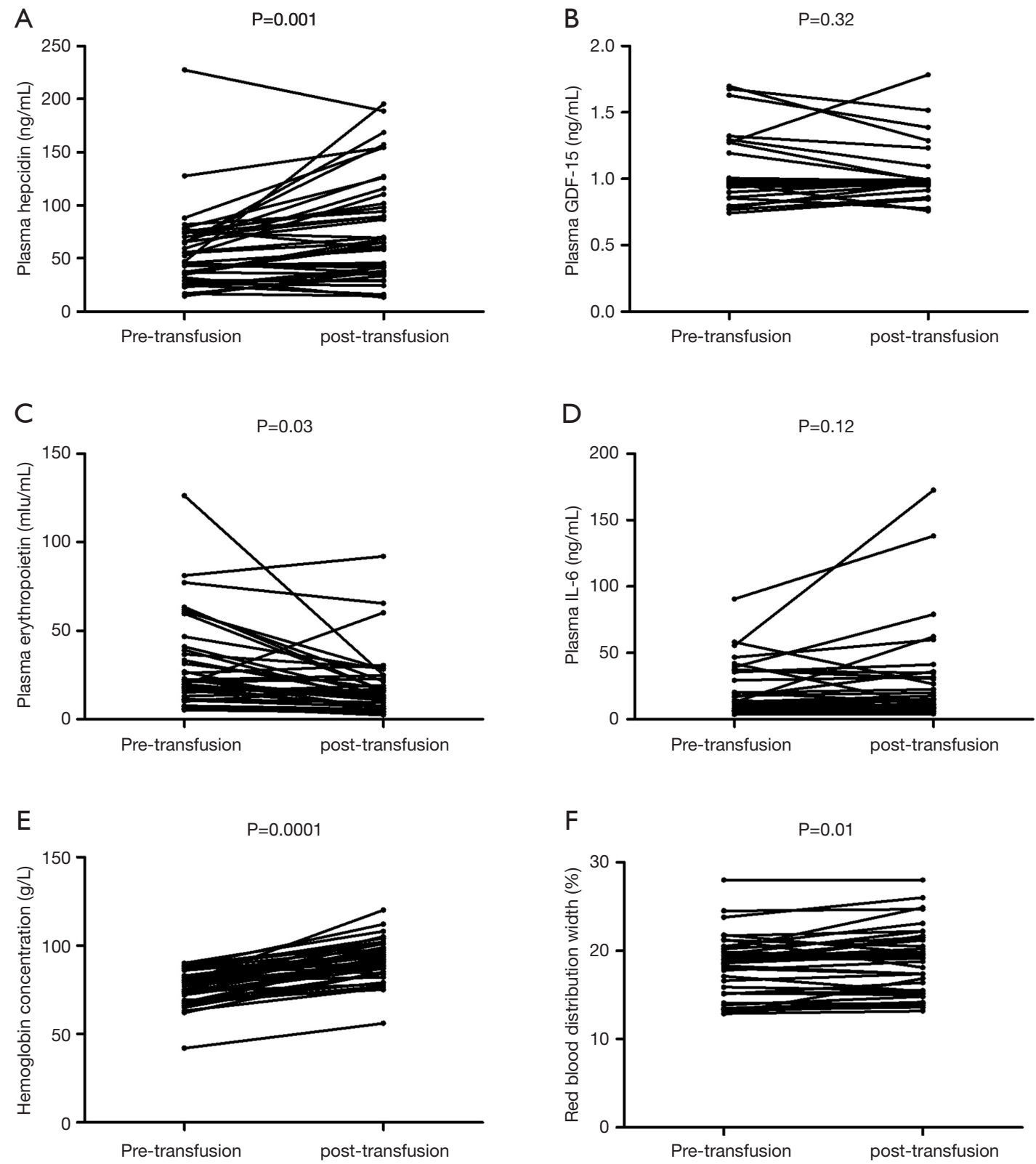

Figure 2 Red blood transfusion results in changes in plasma parameters. (A) Plasma hepcidin (55.79 \pm 36.67 vs. 77. 57 $\pm 49.86, \mathrm{P}=0.001)$. (B) Plasma GDF-15 (1.04 \pm 0.23 vs. $1.02 \pm 0.19, \mathrm{P}=0.32)$. (C) Plasma erythropoietin (28.76 \pm 25.93 vs. 17.86 \pm 18.13 , P=0.03). (D) Plasma IL-6 (19.16 \pm 18.84 vs. $24.97 \pm 35.06, \mathrm{P}=0.12)$. (E) Hemoglobin level $(76.83 \pm 9.68$ vs. $92.18 \pm 11.59, \mathrm{P}=0.0001)$. (F) Red blood cell distribution width (17.94 \pm 3.53 vs. $18.64 \pm 3.77, \mathrm{P}=0.01)$.

post-transfusion. A significant increase of $\mathrm{Hb}$ level after transfusion was shown in all patients. The plasma hepcidin increased after transfusion, and the difference between pre-transfusion and post-transfusion was statistically significant as revealed by the paired $t$-test $(\mathrm{P}=0.001)$. After the transfusion, plasma erythrogenin had decreased to baseline values. RBC transfusion resulted in a decrease in plasma erythropoietin $(\mathrm{P}=0.03)$. Plasma IL-6 $(\mathrm{P}=0.12)$ and GDF-15 ( $\mathrm{P}=0.32)$ showed no significant change after transfusion. RDW was significantly increased after transfusion $(\mathrm{P}=0.01)$.

The post-transfusion changes (\%) of variables after 
Table 2 Post-transfusion change of variables in metastasis(+) and metastasis(-) patients

\begin{tabular}{|c|c|c|c|}
\hline Variables & \multicolumn{2}{|c|}{$\%$ change } & $\mathrm{P}$ \\
\hline Hepcidin & $41.13 \pm 35.95$ & $45.76 \pm 83.32$ & 0.84 \\
\hline GDF-15 & $-3.00 \pm 15.32$ & $0.37 \pm 9.97$ & 0.40 \\
\hline IL-6 & $8.71 \pm 43.38$ & $35.51 \pm 93.28$ & 0.30 \\
\hline Hemoglobin & $19.90 \pm 12.80$ & $20.99 \pm 10.73$ & 0.77 \\
\hline Hematokrit & $9.49 \pm 29.54$ & $18.18 \pm 9.45$ & 0.18 \\
\hline Red cell distribution width & $3.74 \pm 7.94$ & $4.39 \pm 9.17$ & 0.82 \\
\hline
\end{tabular}

GDF-15, growth differentiation factor 15; IL-6, interleukin 6.

Table 3 Post-transfusion change of variables in transfusion volume 2 units and 4 units patients

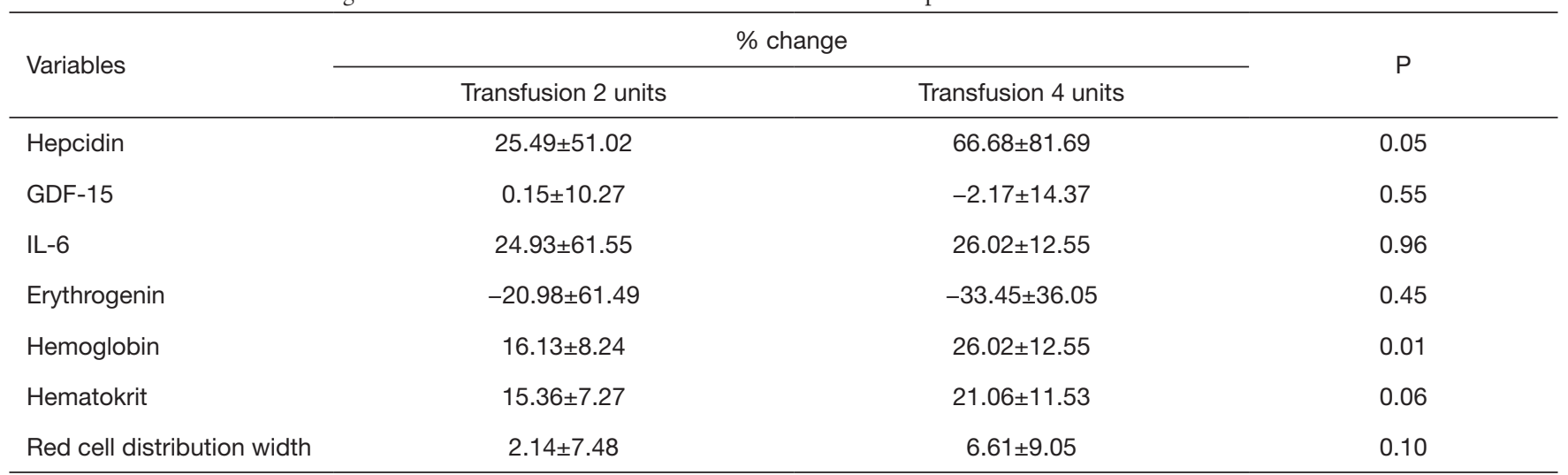

GDF-15, growth differentiation factor 15; IL-6, interleukin 6.

transfusion compared to baseline in metastatic compared to non-metastatic patients are shown in Table 2. Variables did not show a different effect of RBC transfusion in metastatic compared to non-metastatic patients.

Table 3 illustrates the changes (\%) of variables after transfusion between blood transfusion volume $2 \mathrm{U}$ and $4 \mathrm{U}$ group. The change (\%) of Hb level was significantly different between the two groups. Plasma hepcidin of patients in the two groups showed an increase, and the change (\%) of hepcidin in the $4 \mathrm{U}$ group was more than the $2 \mathrm{U}$ group. Other variables did not show a different effect of $\mathrm{RBC}$ transfusion in the $2 \mathrm{U}$ group compared to the $4 \mathrm{U}$ group.

\section{Discussion}

To our knowledge, this is the first study to investigate the effect of RBC transfusion on plasma hepcidin and GDF-15 in gastric cancer patients. The main findings of this study were that (I) the levels of plasma hepcidin and GDF-15 in metastasis cancer patients were higher than those in nonmetastasis cancer patients; (II) RBC transfusion increased plasma hepcidin and decreased plasma erythropoietin; (III) RBC transfusion resulted in an increase in hepcidin levels in patients with transfusion volume $4 \mathrm{U}$ compared to $2 \mathrm{U}$.

The present study showed that high plasma hepcidin and GDF-15 levels accompanied metastasis. This result is in line with previous studies. From previous studies, it is clear that elevated hepcidin and GDF-15 are not specific to a particular metastasis cancer type. Elevated serum hepcidin was found in various metastasized cancers rather than non-metastasis, including prostate cancer, breast cancer, colon cancer, and non-small cell lung cancer $(24,34,35)$. The high level of serum hepcidin contributes to reduced 
ferroportin levels, and low ferroportin levels result in iron accumulation in cancer cells, which in turn promotes cancer cell proliferation and metastasis. Vocka et al. showed that GDF-15 was a useful biomarker in patients with metastatic colorectal cancer (31). Elevated Plasma GDF-15 was indicated to be associated with lymph node metastases (36). Studies have indicated that the serum GDF-15 is correlated with systemic inflammation in advanced gastric cancer, such as IL-1 and TNF-a, and produced by activated macrophages (37). Cancer is also known to be accompanied by systemic inflammation. Hansen et al. reported an association between systemic inflammation and metastasis $(38,39)$, while Li et al. demonstrated that GDF15 could activate Smad2 by binding to TGF- $\beta$ receptors to promote the development of EMT and increase the metastasis of colon cancer (40).

In our study, an increase in plasma hepcidin concentration after RBC transfusion was observed. This result confirms our hypothesis. To explore the reasons for this increase, plasma erythropoietin and IL-6 were analyzed. A significant difference in changes of erythropoietin level was found in present study, which is consistent with the aforementioned studies (33). The results can be attributed to RBC transfusion improving hemoglobin level and ameliorating anemia, and, as a consequence, suppressing the production of erythropoietin and increasing hepcidin concentration, especially in the short term after transfusion. There were no changes in plasma IL-6. We believe it was already higher in cancer patients than in the healthy group, and the effect of transfusion was negligible. Hult et al. found a similar result in that there were no signs of IL-6 production up to $48 \mathrm{~h}$ after transfusion (41). Meanwhile, transfusion did not affect plasma GDF-15 in the present study. We think that the unchanged inflammatory factors after blood transfusion are one of the reasons for this.

In this study, an additional finding was that the RDW of patients increased after transfusion. The link between $\mathrm{RBC}$ distribution and transfusion was first reported by Savino and his colleagues in critically ill patients (42). In previous studies, RDW was identified as having prognostic value in cancer patients (43). Montagnana summarized the correction between RDW and cancer and suggested that RDW might have a diagnostic and prognostic role in various cancers (44). Elevated RDW after transfusion could be one of the factors that lead to poor prognosis of cancer patients after blood transfusion.

Also, we found that the change (\%) of plasma hepcidin was associated with blood transfusion volume. The change
(\%) in blood transfusion volume in the $>2 \mathrm{U}$ group was more than the $\leq 2 \mathrm{U}$ group. However, the change (\%) of plasma erythrogenin was not different in the two groups. Except for the decrease of erythrogenin, there was another mechanism on the link between the change (\%) of plasma hepcidin and transfusion volume.

There are some limitations to this study. Owing to insufficient blood supply during the study period, we failed to investigate the association between the changes of hepcidin and the different stored period of packed $\mathrm{RBC}$ transfusion. The numbers of eligible patients were inadequate, but it was the largest sample we could obtain.

\section{Conclusions}

In conclusion, this study showed that RBC transfusion could increase hepcidin concentration and have no effect on plasma GDF-15 in gastric cancer patients. It is well known that $\mathrm{RBC}$ transfusion is an independent factor for prognosis in cancer patients. As previous studies have shown, high hepcidin level is associated with metastasis, TNM stage, and prognosis. The association between increased hepcidin concentration and the adverse effect of RBC transfusion deserves more attention in future studies.

\section{Acknowledgments}

Funding: This study was supported by the Fujian Provincial Health Technology Project (Grant number: 2016-ZQN19), the National Clinical Key Specialty Construction Program of China and Science and Technology Program of Fujian Province (Grant number: 2018Y2003).

\section{Footnote}

Conflicts of Interest: The authors have no conflicts of interest to declare.

Ethical Statement: The authors are accountable for all aspects of the work in ensuring that questions related to the accuracy or integrity of any part of the work are appropriately investigated and resolved. This study was approved by the Ethical Committee of Fujian Cancer Hospital (KT2018-013-01).

\section{References}

1. Chen $W$, Zheng R, Baade PD, et al. Cancer statistics in 
China, 2015. CA Cancer J Clin 2016;66:115-32.

2. Wasada I, Eguchi H, Kurita M, et al. Anemia affects the quality of life of Japanese cancer patients. Tokai J Exp Clin Med 2013;38:7-11.

3. Doni L, Perin A, Manzione L, et al. The impact of anemia on quality of life and hospitalisation in elderly cancer patients undergoing chemotherapy. Crit Rev Oncol Hematol 2011;77:70-7.

4. Kanuri G, Sawhney R, Varghese J, et al. Iron Deficiency Anemia Coexists with Cancer Related Anemia and Adversely Impacts Quality of Life. PLoS One 2016;11:e0163817.

5. Obermair A, Cheuk R, Horwood K, et al. Impact of hemoglobin levels before and during concurrent chemoradiotherapy on the response of treatment in patients with cervical carcinoma: preliminary results. Cancer 2001;92:903-8.

6. Wei YS, Hong CY, Zhao CX, et al. Clinicopathological features and prognosis of colorectal cancer patients with preoperative cancer-related anemia. Zhonghua Wei Chang Wai Ke Za Zhi 2012;15:385-7.

7. Xiao H, Quan H, Pan S, et al. Impact of peri-operative blood transfusion on post-operative infections after radical gastrectomy for gastric cancer: a propensity score matching analysis focusing on the timing, amount of transfusion and role of leukocyte depletion. J Cancer Res Clin Oncol 2018;144:1143-54.

8. Xue L, Chen XL, Wei-Han Z, et al. Impact of Perioperative Blood Transfusion on Postoperative Complications and Prognosis of Gastric Adenocarcinoma Patients with Different Preoperative Hemoglobin Value. Gastroenterol Res Pract 2016;2016:6470857.

9. Squires MH 3rd, Kooby DA, Poultsides GA, et al. Effect of Perioperative Transfusion on Recurrence and Survival after Gastric Cancer Resection: A 7-Institution Analysis of 765 Patients from the US Gastric Cancer Collaborative. J Am Coll Surg 2015;221:767-77.

10. Xu D, Fang X, Li Y, et al. Perioperative blood transfusion is one of the factors that affect the prognosis of gastric cancer. J BUON 2018;23:672-7.

11. Liu X, Ma M, Huang H, et al. Effect of perioperative blood transfusion on prognosis of patients with gastric cancer: a retrospective analysis of a single center database. BMC Cancer 2018;18:649.

12. Agnes A, Lirosi MC, Panunzi S, et al. The prognostic role of perioperative allogeneic blood transfusions in gastric cancer patients undergoing curative resection: A systematic review and meta-analysis of non-randomized, adjusted studies. Eur J Surg Oncol 2018;44:404-19.

13. Sangkhae V, Nemeth E. Regulation of the Iron Homeostatic Hormone Hepcidin. Adv Nutr 2017;8:126-36.

14. Zuo E, Lu Y, Yan M, et al. Increased expression of hepcidin and associated upregulation of JAK/ STAT3 signaling in human gastric cancer. Oncol Lett 2018;15:2236-44.

15. Pan X, Lu Y, Cheng X, et al. Hepcidin and ferroportin expression in breast cancer tissue and serum and their relationship with anemia. Curr Oncol 2016;23:e24-6.

16. Tesfay L, Clausen KA, Kim JW, et al. Hepcidin regulation in prostate and its disruption in prostate cancer. Cancer Res 2015;75:2254-63.

17. Manz DH, Blanchette NL, Paul BT, et al. Iron and cancer: recent insights. Ann N Y Acad Sci 2016;1368:149-61.

18. Chen Q, Wang L, Ma Y, et al. Increased hepcidin expression in non-small cell lung cancer tissue and serum is associated with clinical stage. Thorac Cancer 2014;5:14-24.

19. Ciniselli CM, De Bortoli M, Taverna E, et al. Plasma hepcidin in early-stage breast cancer patients: no relationship with interleukin-6, erythropoietin and erythroferrone. Expert Rev Proteomics 2015;12:695-701.

20. Guo W, Zhang S, Chen Y, et al. An important role of the hepcidin-ferroportin signaling in affecting tumor growth and metastasis. Acta Biochim Biophys Sin (Shanghai) 2015;47:703-15.

21. Wu XN, Su D, Wang L, et al. Roles of the hepcidinferroportin axis and iron in cancer. Eur J Cancer Prev 2014;23:122-33.

22. Toshiyama R, Konno M, Eguchi H, et al. Association of iron metabolic enzyme hepcidin expression levels with the prognosis of patients with pancreatic cancer. Oncol Lett 2018;15:8125-33.

23. Chua AC, Klopcic B, Lawrance IC, et al. Iron: an emerging factor in colorectal carcinogenesis. World J Gastroenterol 2010;16:663-72.

24. Orlandi R, De Bortoli M, Ciniselli CM, et al. Hepcidin and ferritin blood level as noninvasive tools for predicting breast cancer. Ann Oncol 2014;25:352-7.

25. Nemeth E, Tuttle MS, Powelson J, et al. Hepcidin regulates cellular iron efflux by binding to ferroportin and inducing its internalization. Science 2004;306:2090-3.

26. Pak M, Lopez MA, Gabayan V, et al. Suppression of hepcidin during anemia requires erythropoietic activity. Blood 2006;108:3730-5.

27. Liu Q, Davidoff O, Niss K, et al. Hypoxia-inducible factor regulates hepcidin via erythropoietin-induced 
erythropoiesis. J Clin Invest 2012;122:4635-44.

28. Kim A, Nemeth E. New insights into iron regulation and erythropoiesis. Curr Opin Hematol 2015;22:199-205.

29. Cotte J, Collombel C, Carron R. Study of the action of an inhibitor or histidine decarboxylase on the metabolism of histidine. Sem Ther 1966;42:595-601.

30. Liu JY, Dong XX, Lu JN, et al. Utility of GDF-15 as a diagnostic biomarker in gastric cancer: an investigation combining GEO, TCGA and meta-analysis. FEBS Open Bio 2018;9:35-42.

31. Vocka M, Langer D, Fryba V, et al. Growth/differentiation factor 15 (GDF-15) as new potential serum marker in patients with metastatic colorectal cancer. Cancer Biomark 2018;21:869-74.

32. Lorenz L, Muller KF, Poets CF, et al. Short-Term Effects of Blood Transfusions on Hepcidin in Preterm Infants. Neonatology 2015;108:205-10.

33. Pasricha SR, Frazer DM, Bowden DK, et al. Transfusion suppresses erythropoiesis and increases hepcidin in adult patients with beta-thalassemia major: a longitudinal study. Blood 2013;122:124-33.

34. Traeger L, Ellermann I, Wiethoff H, et al. Serum Hepcidin and GDF-15 levels as prognostic markers in urothelial carcinoma of the upper urinary tract and renal cell carcinoma. BMC Cancer 2019;19:74.

35. Kamai T, Tomosugi N, Abe H, et al. Increased serum hepcidin-25 level and increased tumor expression of hepcidin mRNA are associated with metastasis of renal cell carcinoma. BMC Cancer 2009;9:270.

36. Staff AC, Trovik J, Eriksson AG, et al. Elevated plasma

Cite this article as: Liu J, Chen S, Ye X. The effect of red blood cell transfusion on plasma hepcidin and growth differentiation factor 15 in gastric cancer patients: a prospective study. Ann Transl Med 2019;7(18):466. doi: 10.21037/ atm.2019.08.33 growth differentiation factor-15 correlates with lymph node metastases and poor survival in endometrial cancer. Clin Cancer Res 2011;17:4825-33.

37. Lu Z, Yang L, Yu J, et al. Change of body weight and macrophage inhibitory cytokine-1 during chemotherapy in advanced gastric cancer: what is their clinical significance? PLoS One 2014;9:e88553.

38. Hansen MT, Forst B, Cremers N, et al. A link between inflammation and metastasis: serum amyloid A1 and A3 induce metastasis, and are targets of metastasis-inducing S100A4. Oncogene 2015;34:424-35.

39. Vincent CT, Fuxe J. EMT, inflammation and metastasis. Semin Cancer Biol 2017;47:168-9.

40. Li C, Wang J, Kong J, et al. GDF15 promotes EMT and metastasis in colorectal cancer. Oncotarget 2016;7:860-72.

41. Hult A, Malm C, Oldenborg PA. Transfusion of cryopreserved human red blood cells into healthy humans is associated with rapid extravascular hemolysis without a proinflammatory cytokine response. Transfusion 2013;53:28-33.

42. Spadaro S, Taccone FS, Fogagnolo A, et al. The effects of blood transfusion on red blood cell distribution width in critically ill patients: a pilot study. Transfusion 2018;58:1863-9.

43. Song Y, Huang Z, Kang Y, et al. Clinical Usefulness and Prognostic Value of Red Cell Distribution Width in Colorectal Cancer. Biomed Res Int 2018;2018:9858943.

44. Montagnana M, Danese E. Red cell distribution width and cancer. Ann Transl Med 2016;4:399. 